\title{
Optimizing the restored chemotactic behavior of anticancer agent Salmonella enterica serovar Typhimurium VNP20009
}

\author{
Katherine M. Broadway ${ }^{\mathrm{a}, 1}$, Seungbeum Suh ${ }^{\mathrm{b}, 1}$, Bahareh Behkam ${ }^{\mathrm{b}, \mathrm{c}}$, Birgit E. Scharf ${ }^{\mathrm{a}, *}$ \\ a Department of Biological Sciences, Life Sciences I, Virginia Tech, Blacksburg, VA 24061, USA \\ b Department of Mechanical Engineering, Kelly Hall, Virginia Tech, Blacksburg, VA 24061, USA \\ ${ }^{\mathrm{c}}$ School of Biomedical Engineering and Sciences, Virginia Tech, Blacksburg, VA 24061, USA
}

\section{A R T I C L E I N F O}

\section{Keywords:}

Bacterial anti-tumor therapy

Chemotaxis

Microfluidic chemotaxis assay

Tumor-colonizing bacteria

\begin{abstract}
A B S T R A C T
Bacteria, including strains of Salmonella, have been researched and applied as therapeutic cancer agents for centuries. Salmonella are particularly of interest due to their facultative anaerobic nature, facilitating colonization of differentially oxygenated tumor regions. Additionally, Salmonella can be manipulated with relative ease, resulting in the ability to attenuate the pathogen or engineer vectors for drug delivery. It was recently discovered that the anti-cancer Salmonella enterica serovar Typhimurium strain VNP20009 is lacking in chemotactic ability, due to a non-synonymous single nucleotide polymorphism in cheY. Replacing the mutated copy of cheY with the wild-type sequence restored chemotaxis to $70 \%$ of the parental strain. We aimed to investigate further if chemotaxis of VNP20009 can be optimized. By restoring the gene $m s b B$ in VNP20009 che $\mathrm{Y}^{+}$, which confers attenuation by lipid A modification, we observed a $9 \%$ increase in swimming speed, $13 \%$ increase in swim plate performance, $19 \%$ increase in microfluidic device partitioning towards the attractant at the optimum concentration gradient, and mitigation of a non-motile cell subpopulation. We conclude that chemotaxis can be enhanced further but at the cost of changing one defining characteristic of VNP20009. A less compromised strain might be needed to employ for investigating bacterial chemotaxis in tumor interactions.
\end{abstract}

\section{Introduction}

Bacteria have been researched for their potential application as therapeutic agents for centuries. Specifically, the enterobacterium Salmonella is an attractive model for studying tumor targeting due to its facultative anaerobic nature, allowing for colonization of the differentially oxygenated necrotic and viable tumor regions (Leschner and Weiss, 2010a), as well as large and small tumors (Pawelek et al., 2003). In addition, Salmonella can be manipulated genetically with relative ease and possesses an intriguing facultative intracellular lifestyle (Nallar et al., 2017). Importantly, an attenuated, auxotrophic Salmonella enterica serovar Typhimurium strain has resulted in decreased tumor growth in mice bearing B16F10 melanoma and has been suggested to be able to colonize solid tumors up to a reported 9000 times greater than the liver (Pawelek et al., 1997).

Several strains of $S$. Typhimurium have been constructed for the purpose of tumor targeting and chemotherapy delivery, most mentionable being: A1 (Zhao et al., 2005)/A1-R (Zhao et al., 2007; Hiroshima et al., 2013; Hoffman, 2016; Hiroshima et al., 2014; Yano et al., 2014; Murakami et al., 2016; Kawaguchi et al., 2016), BRD509/BRD509E
(Yoon et al., 2014; al-Ramadi et al., 2009), $\chi 4550$ (Saltzman et al., 1996), CRC2631 (Eisenstark et al., 2007), $\Delta$ ppGpp (Jiang et al., 2013; Kim et al., 2015; Nguyen et al., 2010), LH340 (Zhang et al., 2007; VanCott et al., 1998; Jia et al., 2012), LVR01 (Grille et al., 2014), MvP728 (Manuel et al., 2011; Xu et al., 2014; Xiong et al., 2010), RE88 (Xiang et al., 2005; Lee et al., 2006; Qian et al., 2011), SA186 (Chirullo et al., 2015), SB824 (Roider et al., 2011), SL3261 (Lin et al., 2012; Ye et al., 2013), SL7207 (Jarosz et al., 2013; Li et al., 2013; Shi et al., 2016; Berger et al., 2013), YB1 (Li et al., 2017; Yu et al., 2015), and VNP20009 (Clairmont et al., 2000). VNP20009 was created by Low et al. (Low et al., 2004) from strain 14028s through selection for hyperinvasion by chemical/UV mutagenesis. The strain additionally contains two targeted deletions resulting in purine auxotrophy ( $p u r M^{-}$), and attenuation by modification of lipid $\mathrm{A}\left(m s b B^{-}\right.$) (Low et al., 2004). VNP20009 was assessed in a variety of animal models, for acute toxic effects in cynomolus monkeys, yorkshire pigs and mice (Lee et al., 2000), as well as distribution in mice and nonhuman primates (Clairmont et al., 2000). VNP20009 was found to preferentially target tumors in mice, with tumor:normal tissue colonization ratios of $\geq 1000: 1$ (Clairmont et al., 2000). Finally, VNP20009 has been

\footnotetext{
* Corresponding author at: Biological Sciences, Life Sciences I Virginia Tech Blacksburg, VA 24061, USA. Tel.: +1540 2310757 ; fax: +1 5402314043.

E-mail address: bscharf@vt.edu (B.E. Scharf).

${ }^{1}$ These authors equally contributed to the work.
} 
attributed to curing a dog with epitheliotropic lymphoma, in which the patient received two infusions of the bacteria, experienced a greater than $50 \%$ reduction of the tumor, and after five total infusions was disease free (Thamm et al., 2005). These studies culminated in a 2001 Phase 1 Clinical Trial, where VNP20009 was introduced as a treatment to patients with nonresponsive metastatic melanoma or renal cell carcinoma. Although colonization was observed for some patients, treatment with VNP20009 did not result in tumor regression (Toso et al., 2002). The optimized balance of safe delivery and therapeutic efficacy remains a topic of interest for future use of Salmonella as a cancer therapy.

Many $S$. Typhimurium traits have been investigated for optimization of bacterial localization and retardation of tumor growth. These comprise components of virulence including pathogenicity islands SPI-1 and SPI-2, motility, chemotaxis, biofilm formation and metabolism (Crull et al., 2011a; Crull et al., 2011b; Chorobik et al., 2013). Utilization of chemotaxis is particularly an interesting concept, because the machinery can be manipulated to facilitate bacterial colonization of specified regions of tumors based on the chemoattractant composition. Generally, it has been found that bacterial chemotaxis is favorable for tumor spheroid colonization in vitro, with specific chemoreceptors facilitating tumor microenvironment localization (Kasinskas and Forbes, 2006; Kasinskas and Forbes, 2007). In contrast, in immunocompetent mice, non-chemotactic or non-motile Salmonella strains colonized tumors with the same efficiency as the wild type (Crull et al., 2011a; Stritzker et al., 2010; Leschner and Weiss, 2010b). Recently however, in a high-throughput screening of mutant $S$. Typhimurium strains, the presence of chemotaxis gene cheY and motility genes motA and $\operatorname{mot} B$ was found to hold an advantage for tumor colonization (Silva-Valenzuela et al., 2016). Thus, due to different experimental conditions, $S$. Typhimurium strains and cancer models, the role of chemotaxis in vivo is controversial, or perhaps greatly context dependent.

VNP20009 was recently discovered to be deficient in chemotaxis, due to a non-synonymous single nucleotide polymorphism (SNP) in the gene encoding the chemotaxis two-component response regulator, cheY (Broadway et al., 2015). Upon replacing the deficient copy of cheY with the wild-type copy, chemotaxis was recovered to $70 \%$ of the parental strain (Broadway et al., 2015). The overall objective of this study was to determine if chemotaxis of VNP2009 can be optimized to enable future analysis of maximal tumor targeting potentially through positive chemotaxis to metabolic byproducts of cancerous tissue. We report that by restoring the gene which encodes a lipid A altering enzyme $m s b B$, we significantly increased the performance of the strain in chemotaxis assays, including swim plates and a microfluidic device, which establishes attractant gradients. We discovered that the enhancement in population scale chemotaxis performance was partially due to an increase in the motile cell population of VNP20009 $\mathrm{cheY}^{+}$upon curing the $m s b B$ defect. In conclusion, lack of modified lipid A due to the $m s b B$ deletion of VNP20009, which is a defining characteristic by the National Cancer Drug Dictionary, yielding the strain's safety as an anticancer therapy, also negatively impacts chemotaxis, which makes it a less ideal strain for exploring the impact of bacterial chemotaxis in cancer colonization.

\section{Materials and methods}

\subsection{Bacterial strains and growth conditions}

Bacterial cell cultures were routinely grown in MSB (Murray et al., 2001) medium ( $1 \%$ tryptone, $0.5 \%$ yeast extract, $2 \mathrm{mM} \mathrm{MgSO}_{4}, 2 \mathrm{mM}$ $\mathrm{CaCl}_{2}$ ) at $37^{\circ} \mathrm{C}$.

\subsection{Mutant construction}

Mutant strains were constructed using the lambda-red genetic engineering system (Karlinsey, 2007; Datsenko and Wanner, 2000). The $m s b B$ gene in VNP20009 $c h e Y^{+}$was restored by replacing the mutated copy with the parental copy. We were careful in this construction, not to alter the $3^{\prime}$ extension of pykA in VNP20009, which resulted in 13 new codons (Broadway et al., 2014). This was ensured using primer pairs msbB400dn with MID1dn, and msbB400up with MID2up, with template chromosomal DNA from VNP20009 or 14028, respectively (see Supplemental Table 1). Each of the MID primers have overlapping regions, resulting in a DNA fragment approximately 1.7 kbps in size.

After deleting $m s b B$ in strain 14028 , a mutant carrying the Suwwan deletion was selected for by plating on LB-0 plates (lysogeny broth (Bertani, 1951) without $\mathrm{NaCl}$ ) supplemented with $6 \mathrm{mM}$ ethylene glycol-bis( $\beta$-aminoethyl ether)-N,N,N',N'-tetraacetic acid (EGTA). All mutations were confirmed by Sanger sequencing.

\subsection{Quantitative swim plate assay}

MSB swim plates $(0.3 \%$ agar $)$ were inoculated with $2.5 \mu \mathrm{L}$ of stationary phase cultures and incubated for approximately $12 \mathrm{~h}$ at $37^{\circ} \mathrm{C}$. Data was normalized to the average of 14028 and represents six experiments, except the negative control VNP20009 with three experiments, each in triplicate.

\subsection{Microfluidic device fabrication and testing}

The design and fabrication of the device was previously described in detail (Traore and Behkam, 2013). Briefly, polyethylene glycol diacrylate (PEG-DA, $\mathrm{MW}=700 \mathrm{Da}, 10 \%(\mathrm{v} / \mathrm{v})$ in phosphate buffered saline (PBS)) solution mixed with $0.5 \%(\mathrm{w} / \mathrm{v})$ of the photoinitiator Irgacure ${ }^{\circledast}$ 2959 (Sigma-Aldrich, St. Louis, MO) was photo-polymerized by $15 \mathrm{sec}-$ onds of UV treatment $\left(365 \mathrm{~nm}, 18 \mathrm{~W} / \mathrm{cm}^{2}\right.$, Omnicure S1000, Vanier, Quebec) within a polydimethylsiloxane (PDMS) enclosure on the glass slide. A three-channel pattern blocked the UV penetration to establish hydrogel wall around the channels. A PDMS layer with access ports for inlet and outlet tubing was placed on the PEG-DA hydrogel layer and the entire assembly was sandwiched between two plexiglas supports to ensure complete seal. Continuous flow of motility buffer (MB; $6.4 \mathrm{mM}$ $\mathrm{K}_{2} \mathrm{HPO}_{4}, 3.5 \mathrm{mM} \mathrm{KH} \mathrm{PO}_{4}, 0.1 \mathrm{mM}$ EDTA, $1 \mu \mathrm{M}_{\mathrm{L}}$-methionine, $10 \mathrm{mM}$ DL-lactate, $2 \mathrm{mM} \mathrm{MgSO}_{4}, 2 \mathrm{mM} \mathrm{CaCl}_{2}, \mathrm{pH} 7.0$ ) at $5 \mu \mathrm{l} / \mathrm{min}$ through the left outer channels and L-aspartic acid in $\mathrm{MB}$ in the right outer channel was maintained for $60 \mathrm{~min}$ using a syringe pump (PHD Ultra syringe pump, Harvard apparatus, Holliston, MA). Finite element analysis software package $\mathrm{COMSOL}^{\circledR}$ was used to evaluate the required diffusion time for L-aspartic acid transport through the hydrogel wall to establish a quasi-steady and linear chemical concentration gradients across the central channel. For instance, the optimum chemical concentration gradient of $1.54 \times 10^{-5} \mathrm{M} / \mathrm{mm}$ was achieved within 60 min where the chemo-attractant channel was filled with the L-aspartic acid at a concentration of $10^{-4} \mathrm{M}$. For the computational model, the diffusion coefficient of L-aspartic acid was attained to be $D=1.5 \times 10^{-6} \mathrm{~cm}^{2} / \mathrm{s}$, from previous work using a Franz diffusion cell (Traore and Behkam, 2013). Once the chemical concentration gradient was established through the central channel, the bacteria suspended in MB were manually injected to the central channel. Bacterial biased distribution due to the chemotaxis reached steady state within $15 \mathrm{~min}$.

\subsection{Imaging and data analysis of the chemotaxis response within microfluidic device}

Bacterial biased migration via chemotaxis within the microfluidic device was captured using a Zeiss AxioObserver Z1 inverted microscope equipped with an AxioCam mRM camera and a $20 \times$ objective. The images were analyzed using an image processing algorithm developed in MATLAB to quantify the number of bacteria located either in the left or right half plane of the central channel. The chemotaxis performance 
was quantified using the chemotaxis partition coefficient (CPC) (Traore and Behkam, 2013), a population-metric showing the chemotaxis responsiveness and is defined by

$\mathrm{CPC}=\frac{\mathrm{B}_{\mathrm{r}}-\mathrm{B}_{1}}{\mathrm{~B}_{\mathrm{r}}+\mathrm{B}_{1}}$

where $B_{r}$ is the number of bacteria in the right half plane of the central channel, $B_{1}$ is the number of bacteria in the left half plane of the central channel. When the chemo-effector is located in the right side of a central channel, CPC values of +1 and -1 indicate the strongest attraction or repellence, respectively. Data are representative of an average of $5 \times 10^{5}$ cells/experiment.

\subsection{Image and data analysis of bacterial swimming speed}

Bacterial movement was captured using a Zeiss AxioObserver Z1 inverted microscope equipped with an AxioCam Hsm camera and a $63 \times$ oil immersion objective at a spatial resolution of $0.31 \mu \mathrm{m} /$ pixel and temporal resolution of $30 \mathrm{fps}$. A minimum of 80 bacteria were tracked in each experiment and three independent set of experiments were carried out for each strain. All experiments were conducted at $37^{\circ} \mathrm{C}$. The recorded images were analyzed in ImageJ using the Manual Tracking plug-in tool (NIH, Bethesda, MD). The instantaneous speeds were calculated by dividing the distances travelled during each time increment by the time increment. The average swimming speed was determined through averaging the instantaneous swimming speeds over independent experiments. The percentage of motile bacteria in the total population was quantified using an image processing algorithm developed in MATLAB, which detects the stationary (non-motile) subpopulation. The position of each bacterium was tracked at $30 \mathrm{~s}$ intervals, and bacteria with unchanged coordinates over two consecutive images were deemed non-motile. A total of 18 images (containing approximately 1500 bacteria on average) were analyzed for each experiment, and three independent set of experiments were carried out for each strain.

\subsection{Flagella labeling, fluorescence microscopy and image acquisition}

Cell cultures were grown to an $\mathrm{OD}_{600}$ of 1.0 in MSB medium at $37{ }^{\circ} \mathrm{C}$ with shaking at $100 \mathrm{rpm}$, and labeling was essentially performed as described elsewhere (Turner et al., 2000). Cells were harvested at $1500 \times g$ for $5 \mathrm{~min}$, washed three times in MB, with a final resuspension in $500 \mu \mathrm{L} \mathrm{MB}$. One vial of Cy3 monofunctional dye was dissolved in $100 \mu \mathrm{L}$ MB with $0.0001 \%$ Tween-20 (MBT) and divided amongst $S$. Typhimurium 14028 and VNP20009 $\mathrm{cheY}^{+}$. Cells were labeled by incubation at room temperature with gentle shaking for $60 \mathrm{~min}$. Cells were washed three times in MB to remove excess dye, diluted 10-fold in

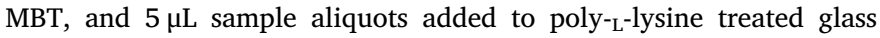
slides. Fluorescently labeled cells and filaments were then observed on an Olympus IX71 microscope with Applied Precision SoftWorx image program, using a $100 \times$ objective. Fluorescence signal was detected using a TRITC filter.

\subsection{Immunoblotting}

Bacterial cell cultures were grown to an $\mathrm{OD}_{600}$ of 1.0 in MSB at $37^{\circ} \mathrm{C}$ with shaking at $100 \mathrm{rpm}$. Culture aliquots of $25 \mu \mathrm{L}$, as well as $25 \mu \mathrm{L}$ supernatant aliquots of a $1 \mathrm{~mL}$ sample centrifuged for $10 \mathrm{~min}$ at $13,000 \times g$, were prepared by the addition of $15 \mu \mathrm{L}$ loading buffer with $\beta$-mercaptoethanol and boiling at $95{ }^{\circ} \mathrm{C}$ for $5 \mathrm{~min}$. Samples were separated in $12.5 \%$ SDS polyacrylamide gel and transferred to a nitrocellulose membrane. The membrane was probed with polyclonal rabbit antibody for Salmonella anti-FliC/FljB $\left(\right.$ Difco $\left.^{\mathrm{TM}}\right)$ at a dilution of 1:50,000. After removal of residual unbound antibody, blots were incubated with donkey anti-rabbit horse radish peroxidase conjugated antibody at a dilution of 1:2500. After removal of unbound antibody, detection was achieved by enhanced chemiluminescence (Amersham

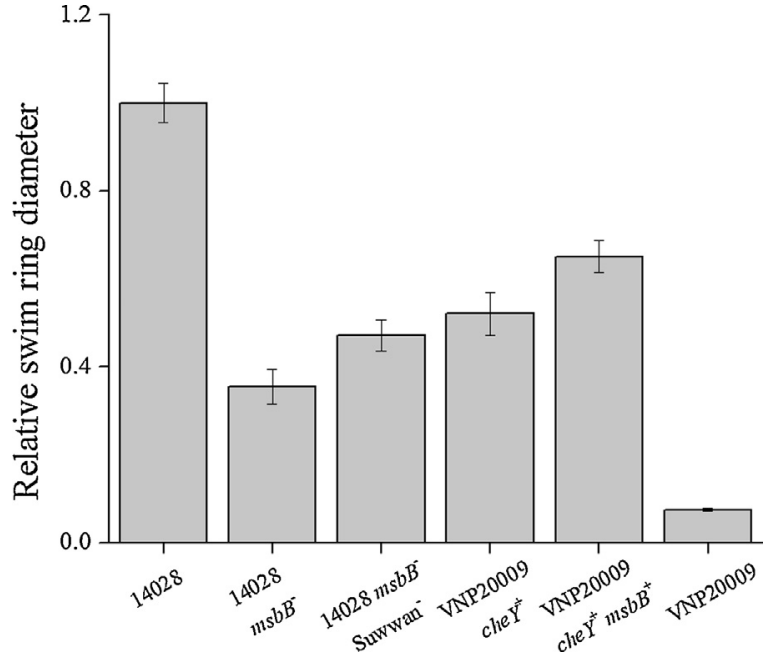

Fig. 1. Chemotactic performance of $S$. Typhimurium wild-type and mutant strains on swim plate. All swim ring diameters were normalized to the average of 14028 , with data representing the average of six experiments, each in triplicate and error bars representing standard deviations. The behavior of all strains is statistically different from each other, as determined by a one-way analysis of variance (ANOVA), using the Tukey-Kramer HSD test for means comparison, with a significance level of 0.05 . All strains are significantly different from each other $(p<0.01)$.

ECL Western Blotting Detection Kit) using Hyperfilm ECL (GE Healthcare). Films were scanned with an Epson Perfection 1640SU and scans were analyzed using ImageJ.

\section{Results}

\subsection{Chemotactic ability is significantly increased by restoring $m s b B$ in} VNP20009 che $^{+}$

We first aimed to determine the contribution of $m s b B$ to the remaining chemotaxis defect observed in VNP20009 $\mathrm{che}^{+}$. We therefore restored the $m s b B$ gene in VNP20009 $c h e Y^{+}$using the lambda red protocol (Datsenko and Wanner, 2000). Swim plates were inoculated with bacterial strains and the chemotactic response was measured. We determined that a restoration of $m s b B$ in the VNP20009 $\mathrm{cheY}^{+}$background increased the relative swim plate phenotype by $13 \%$ (Fig. 1). VNP20009 also carries a Suwwan deletion, which suppresses certain phenotypes caused by the $m s b B$ deletion such as its sensitivity to the chelator ethylene glycol-bis( $\beta$-aminoethyl ether)-N,N,N',N'-tetraacetic acid (EGTA) (Low et al., 2016). To differentiate between the contribution of the $m s b B$ and the Suwwan deletion to the chemotaxis phenotype, we created two mutant strains of the parental strain 14028, one carrying a deletion in $m s b B$ and one that is additionally lacking the Suwwan region. We found an $m s b B$ deletion alone had a severe effect on bacterial chemotaxis, with the average phenotype only reaching $34 \%$ of the parental strain. However, when the Suwwan region was deleted additionally, we found a significant increase (by $12 \%$ ) in the chemotaxis phenotype on swim plate (Fig. 1). We hypothesize that the compensating effect of the Suwwan deletion for several physiological characteristics observed in VNP20009 is also associated with its effect on chemotaxis and motility. Interestingly, there is only a small (5\%) but significant difference between $14028 \mathrm{msbB}^{-}$Suwwan $^{-}$and VNP20009 $\mathrm{cheY}^{+}$of $5 \%$ (Fig. 1), with the only genomic differences between the two strains being the purM disruption and SNPs inherently present in VNP20009 (Broadway et al., 2014). Therefore, $m s b B$ and Suwwan deletions majorly contribute to the chemotaxis defect discovered in VNP20009 $\mathrm{cheY}^{+}$. 


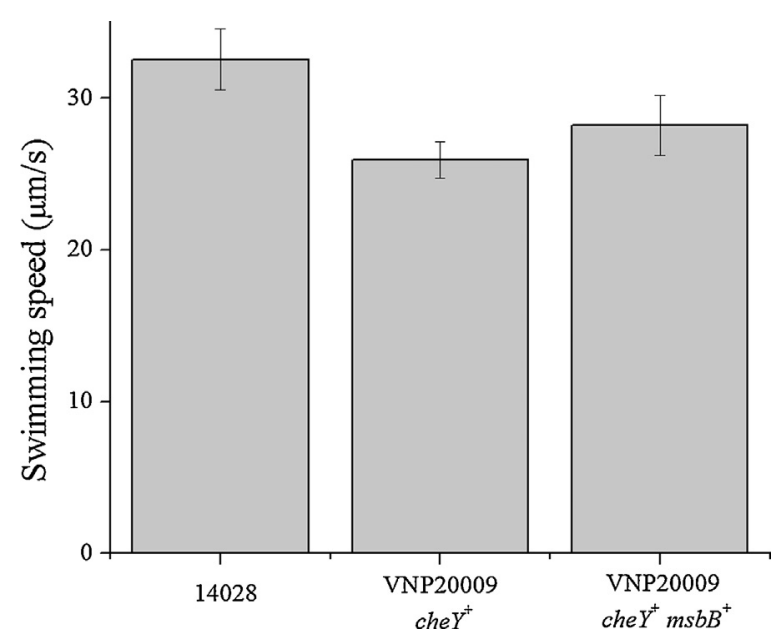

Fig. 2. Swimming speed of $S$. Typhimurium wild-type and mutant strains. The average swimming speed was assessed by 2-D bacterial cell tracking for 14028 ( $n=95)$, VNP20009 $\mathrm{cheY}^{+}(n=67)$, and VNP20009 $\mathrm{cheY}^{+} \mathrm{msbB}^{+}(n=81)$ cells. Error bars represent standard error and statistical significance was determined by a one-way ANOVA followed by Tukey-Kramer HSD test. A p-value of 0.05 was used as the threshold for significance, with statistical differences between all tested strains being $p<0.0001$.

\subsection{Swimming speed is increased by restoration of $m s b B$}

To determine the effect of $m s b B$ on motility, a factor playing into the chemotactic response on swim plates, we measured the swimming speed of 14028, VNP20009 $\mathrm{cheY}^{+}$and VNP20009 $\mathrm{cheY}^{+} \mathrm{msbB}^{+}$ (Fig. 2). The instantaneous speeds from the distances travelled during each time increment were defined as $\frac{\|\Delta \vec{d}\|}{\Delta t}$, where $\Delta d$ is distance travelled and $\Delta t$ is time increment. The average speed of the strain was computed as $\Sigma_{i=1}^{N}\left\|\Delta \overrightarrow{d i}_{i}\right\| / \Delta t / N$, where $N$ is the total number of time steps. The average speed of strains 14028, VNP20009 $\mathrm{cheY}^{+}$, and VNP20009 $\mathrm{cheY}^{+} \mathrm{msbB}^{+}$were $32.5,25.9$, and $28.2 \mu \mathrm{m} / \mathrm{s}$, respectively (Fig. 2), with the measured swimming speed of 14028 being in agreement with the literature (Attmannspacher et al., 2008). There is a $20 \%$ difference in the average swimming speed between 14028 and VNP20009 $\mathrm{cheY}^{+}(p<0.0001)$. Notably, the swimming speed increased by $10 \%$ with the restoration of $m s b B(p<0.0001)$. Therefore, swimming speed might contribute to the reduced chemotaxis proficiency observed in VNP20009 $\mathrm{cheY}^{+}$.

\subsection{A subpopulation of non-motile cells exists in VNP20009 $\mathrm{cheY}^{+}$and is} mitigated by restoration of $m s b B$

While tracking bacteria for swimming speed measurements, we observed a substantial subpopulation of non-motile VNP20009 $\mathrm{cheY}^{+}$ cells, which were not included in the swimming speed calculations. To determine the percentage of motile cells within populations, motile and non-motile cells were manually counted, with bacterial 2-D tracking (Fig. 3). While the whole population of 14028 is motile, only $63 \%$ of the VNP20009 $\mathrm{che}^{+}$cells exhibited motility. Interestingly, the restoration of $m s b B$ resulted in a greater than $20 \%$ increase of the motile population to $83 \%$ in VNP20009 $c h e Y^{+} m s b B^{+}$. Thus, the presence of $m s b B$ considerably affects cell population motility.

\subsection{Flagellation is unaffected by $m s b B$}

The deletion of the $m s b B$ gene could potentially affect flagellation and therefore influence the swimming speed and motility of VNP20009 che $\mathrm{Y}^{+}$. We labeled motile cells with Cy3 fluorescence dye to observe flagella quality for VNP20009 $\mathrm{cheY}^{+}$and 14028. Specifically, we aimed to compare relative number, length, and helical pitch of flagellar filaments. No differences in these three parameters were observed

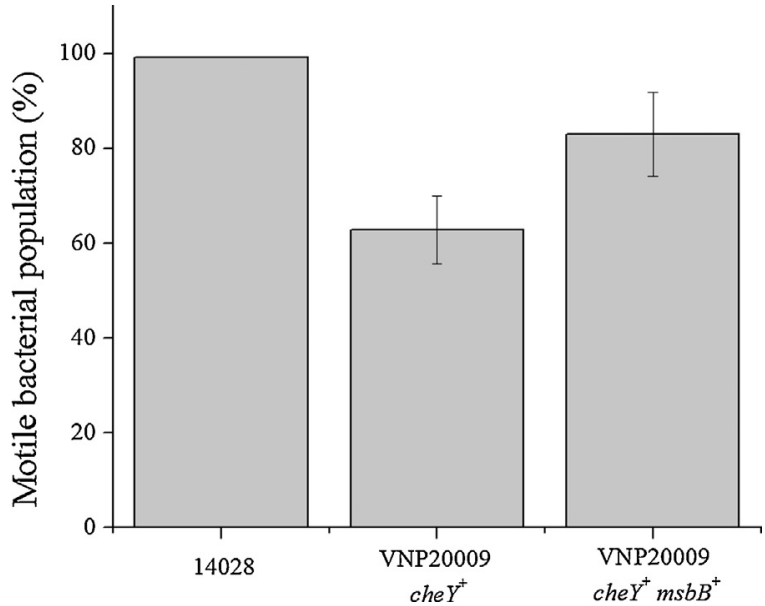

Fig. 3. Proportion of motile cells in $S$. Typhimurium wild-type and mutant strains. The percentage of motile bacteria in the whole cell population was assessed through manual tracking. Data is represented as averages of three independent experiments for each strain, and error bars represent standard error. Statistical significance was determined by a one-way ANOVA followed by Tukey-Kramer HSD test. A p-value of 0.05 was used as the threshold for significance with statistical differences between all tested strains being $p<0.001$.

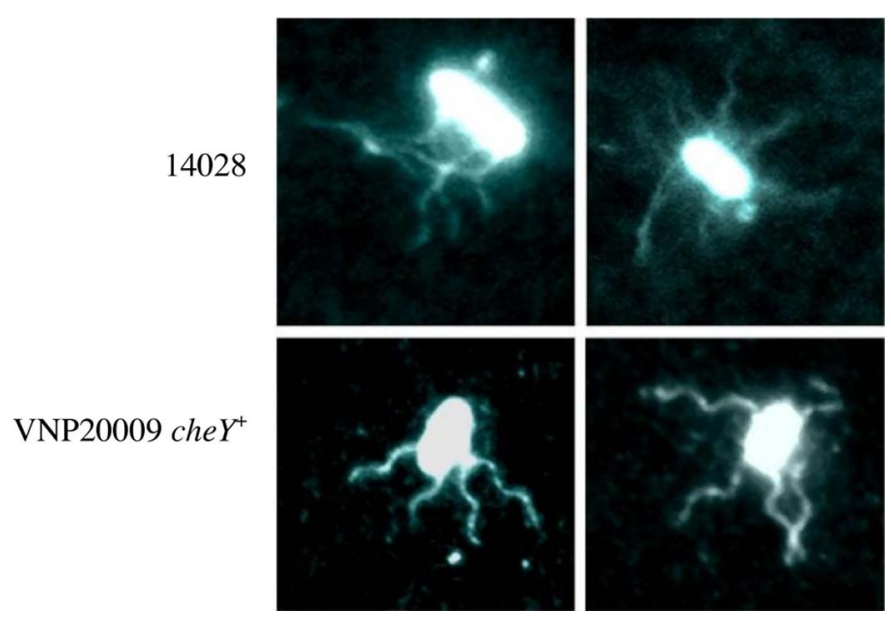

Fig. 4. Representative fluorescence microscopy images of Cy3-labeled $S$. Typhimurium 14028 and VNP20009 $\mathrm{cheY}^{+}$cells.

between the two strains (Fig. 4). However, we noted the presence of free flagellar filament fragments in VNP20009 $\mathrm{che}^{+}$preparations more frequently than in 14028 , which could have been a result of the extensive washing steps required during sample preparation (data not shown). Next, we quantified the amount of flagellin expressed, by probing cell extracts with an antibody against Salmonella FliC/ FljB, compared to a $\mathrm{fliC}^{-}$strain as the negative control. We did not find any differences between 14028, VNP20009 $\mathrm{cheY}^{+}$, and VNP20009 $\mathrm{cheY}^{+}$ $m s b B^{+}$(Fig. 5). The observation of free filaments in the fluorescently labeled VNP20009 $\mathrm{cheY}^{+}$preparations prompted the hypothesis that the $m s b B$ deletion in VNP20009 could result in incomplete support of the flagella through alteration of the LPS/L-ring anchorage system, which might lead to greater flagella shearing. We therefore isolated the culture supernatant after removing bacteria by centrifugation and assayed for presence of flagellin. No differences in the flagellin amounts present in culture supernatants were detected. In fact, statistically, the amount of detected flagellin was not distinguishable between the negative control and test strains. In conclusion, deletion of $m s b B$ does not negatively affect flagellation. 

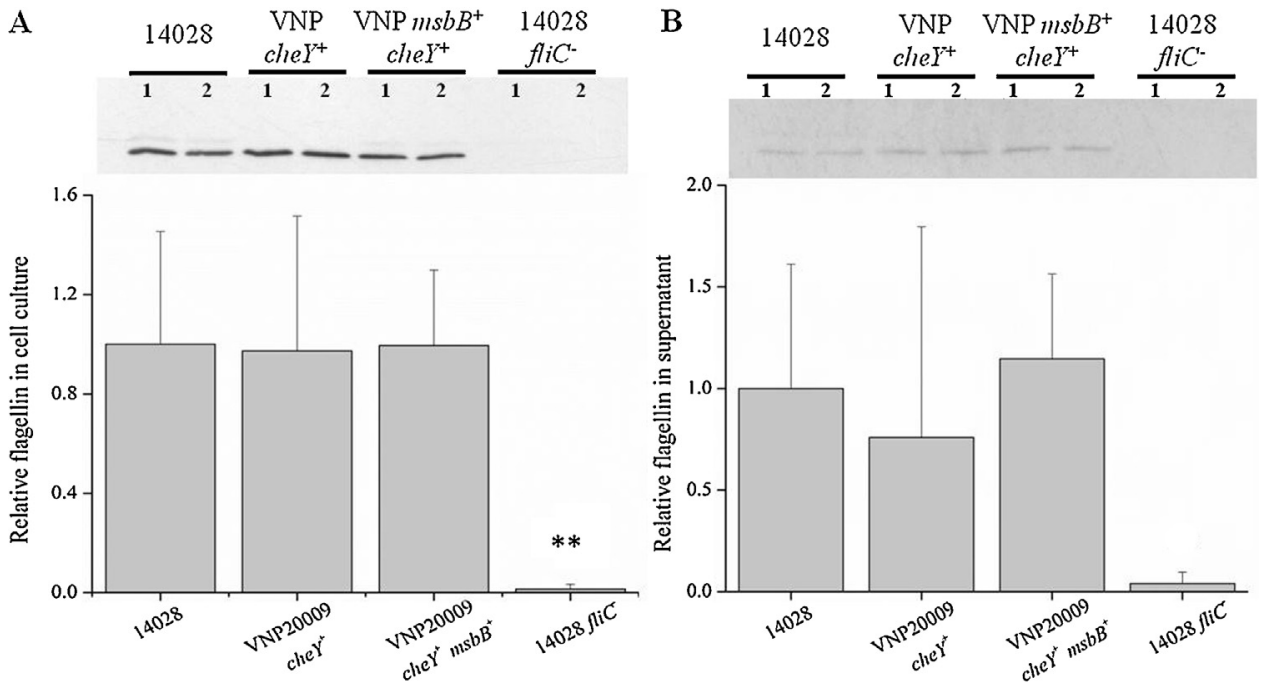

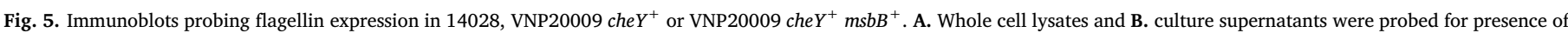

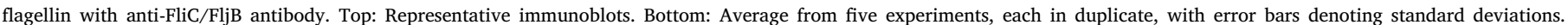

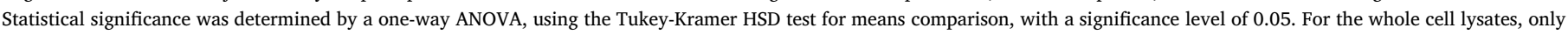

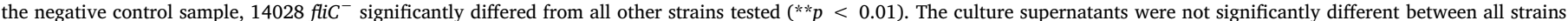
tested.

\subsection{The presence of msbB increases the performance of VNP20009 $\mathrm{cheY}^{+}$ in microfluidic chemotaxis assays}

To investigate the chemoattractant sensitivities of 14028, VNP20009 che $Y^{+}$, and VNP20009 $c h e Y^{+} \mathrm{msbB}^{+}$, we used a microfluidic device. Various concentration gradients ranging from $0 \mathrm{M} / \mathrm{mm}$ (control) to $3.0 \times 10^{-5} \mathrm{M} / \mathrm{mm}$ of L-aspartic acid were established across the central channel of the device to characterize the bacterial response in the presence of the chemo-effector. Continuous flow of Laspartic acid in MB in the right channel and MB in the left channel was maintained to generate static, linear concentration gradients of Laspartic acid via diffusion through the hydrogel walls separating the central channel from the flow channels. The chemotactic partition coefficient (CPC) was computed and shown as a function of concentration gradient, where a value of 1 would indicate the strongest response of bacteria to the chemoattractant (Fig. 6). In control experiments with 14028, VNP20009 $\mathrm{che}^{+}$, and VNP20009 $\mathrm{che}^{+} \mathrm{msbB}^{+}$, bacterial migration did not show a biased distribution, as expected $(|\mathrm{CPC}|<$ 0.05). The attractant concentration gradient eliciting the strongest response was determined to be $1.5 \times 10^{-5} \mathrm{M} / \mathrm{mm}$. As expected, the non-chemotactic parental strain VNP20009 showed no response. While

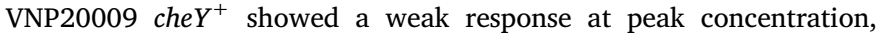
restoration of $m s b B$ resulted in an enhanced response of VNP20009

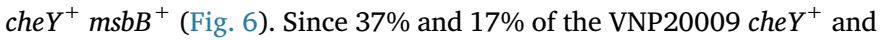
VNP20009 $\mathrm{cher}^{+} \mathrm{msbB}^{+}$cell populations are non-motile, respectively, we analyzed the microfluidic chemotaxis data eliminating non-motile cells. At the optimum concentration gradient, data for the motile population of 14028 and VNP20009 $\mathrm{cheY}^{+} \mathrm{msbB}^{+}$mirror the data acquired for the mixed populations. In contrast, after factoring out the non-motile subpopulation for VNP20009 $\mathrm{che}^{+}$, sensitivity increased by $47 \%$. Thus, the non-motile subpopulation contributes significantly to the chemotactic performance of VNP20009 $\mathrm{cheY}^{+}$in the microfluidic device.

\section{Discussion}

Recent literature has witnessed a comeback for the potential use of bacterial therapy against cancer. Published work on bacterial therapy has increased two fold every 2.5 years since the mid-1990s through 2010 (Forbes, 2010), where engineered strains of Salmonella Typhimurium have been most extensively studied for decades. Success of

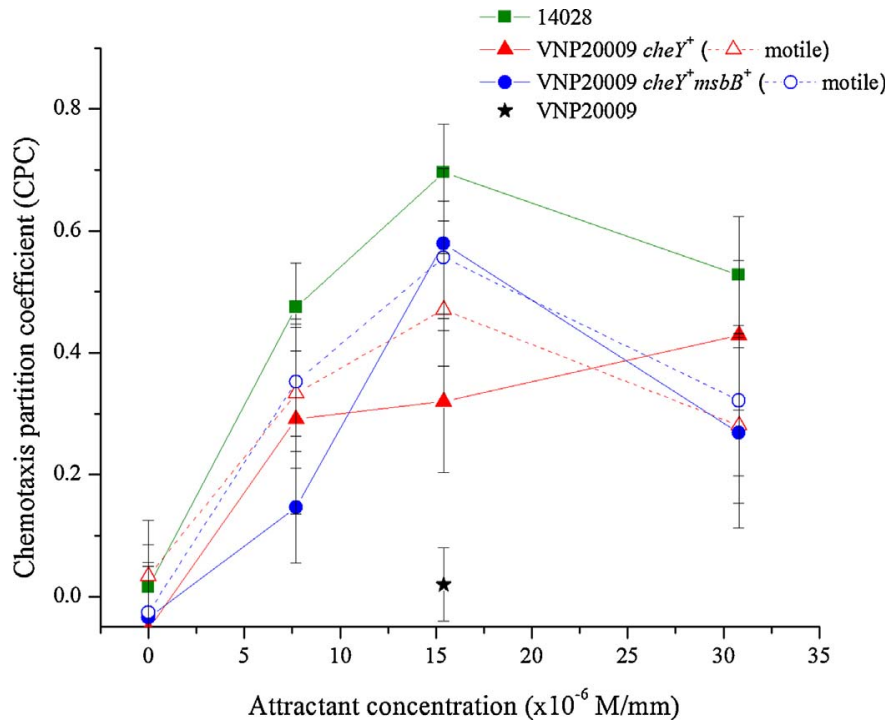

Fig. 6. Chemotactic performance of $S$. Typhimurium wild-type and mutant strains to Laspartic acid in a microfluidic device. Each strain was loaded into the attractant gradient established-microfluidic device and data was collected after $15 \mathrm{~min}$. The CPC value was ascertained for the whole population and only the motile population of each strain, as indicated (the whole population is the motile population for 14028). At the peak attractant concentration, statistical analysis with one-way ANOVA, followed by TukeyKramer HSD test with a significance level of 0.05 , was performed. For motile populations, all strains significantly differed from one another $(p<0.001)$, as well as when whole populations of all strains were compared $(p<0.001)$.

attenuated $S$. Typhimurium strains is well established in diverse tumor and animal models, ranging from immunodeficient to immunocompetent mice; xenograft and syngeneic derived tumors; orthotopic, intravenous, and subcutaneous injection of cancer cells; route of bacterial delivery being intravenous, subcutaneous and oral; with outcomes ranging from tumor growth reduction, metastasis reduction, prolonged survival of mice and even being synergistically with cancer compromising the health of the animal. Efficacy of bacterial strains have been evaluated in preclinical and clinical trials, where the recent 2014 Phase 1 Clinical Trial of $S$. Typhimurium $\chi 4550$ expressing IL-2 was orally administered to patients with unresectable hepatic metastases from a solid tumor (NCT01099631). Despite these impressive strides towards 
the use of bacteria as anticancer biotherapeutics, optimal targeting and anticancer effects of bacterial strains are yet to be achieved beyond preclinical studies.

Previously, we established that the anticancer agent VNP20009 is deficient in chemotaxis, due to a SNP in $c h e Y$, the gene coding for the response regulator in the two component chemotaxis system. Upon replacing the mutated copy of cheY with the 14028 parental copy, chemotaxis was restored to almost $70 \%$, determined using traditional capillary assays (Broadway et al., 2015). Here, we explored several factors to explain the remaining differences in chemotaxis between VNP20009 and the parental strain, including swimming speed, flagellation, attractant sensitivity, and the contribution of $m s b B$ and Suwwan deletion to the remaining defect. The gene $m s b B$ encodes an enzyme that adds terminal myristyl groups to lipid A (Somerville et al., 1996). Without the LPS modifying enzyme, strains of Salmonella are known to possess growth defects as well as EGTA and galactose-MacConkey medium sensitivity (Murray et al., 2001). In strain S. Typhimurium 14028 s, these effects are partially suppressed by the Suwwan deletion, a spontaneous recombination event causing the excision of a 108kilobase region of the genome (Murray et al., 2004). We tested the contribution of this region in conjunction with $m s b B$, through the construction of $14028 \mathrm{msbB}^{-}$Suwwan $^{-}$. Its chemotaxis performance on swim plates was only slightly lower (5\%) than VNP20009 $\mathrm{cheY}^{+}$, which also has the genotype $m s b B^{-}$Suwwan ${ }^{-}$(Fig. 1). This result allows the conclusion that the major contributors to the chemotaxis phenotype of VNP20009 $c h e Y^{+}$result from the $m s b B$ and Suwwan deletions. This result is contrary to previously reported chemotaxis assays of 14028 $m s b B^{-}$, where no significant difference was observed between the $14028 m s b B$ mutant and 14028 wild type (Frahm et al., 2015). A possible explanation for our result is that we measured swim ring diameters after $12 \mathrm{~h}$, whereas in Frahm et al. (Frahm et al., 2015), the measured time point was at $4 \mathrm{~h}$.

Since bacterial metabolism, chemotaxis, and motility are contributing to the swim ring phenotype, we determined the swimming speed of 14028, VNP20009 che $Y^{+}$, and VNP20009 che $Y^{+} \mathrm{msbB}^{+}$. While VNP20009 $\mathrm{che}^{+}$exhibited a $20 \%$ reduction in swimming speed compared to 14028 , restoration of $m s b B$ increased swimming speed by $9 \%$, but not to the level of 14028 (Fig. 2). We therefore conclude that gene restoration increases motility, but this increase might not fully explain the phenomenon we observed on swim plates. We analyzed flagellation and found no difference between flagellar structures of VNP20009 $\mathrm{che}^{+}$and 14028 (Fig. 4) and similar levels of flagellin protein expression of 14028, VNP20009 $\mathrm{che}^{+}$, and VNP20009 $\mathrm{cheY}^{+}$ $\mathrm{msbB}^{+}$(Fig. 5). While flagella shearing was observed for fluorescently labeled VNP20009 $\mathrm{cheY}^{+}$, possibly due to the several centrifugation and resuspension steps involved in the experimental procedure, culture supernatants of VNP20009 strains had no elevated flagellin levels excluding flagella loss during cell culture growth.

Interestingly, we observed a considerably large subpopulation of non-motile VNP20009 $\mathrm{che}^{+}$cells when compared to 14028 and the $m s b B$ restored VNP20009 $c h e Y^{+}$derivative (Fig. 3). Restoration of $m s b B$ in VNP20009 $\mathrm{cheY}^{+}$increased the motile cell population by $20 \%$. The consequences of the presence of a non-motile VNP20009 $\mathrm{cheY}^{+}$ population was most apparent in the microfluidic device experiment. At the optimum concentration gradient, the chemotaxis response to the attractant increased by $47 \%$ when only the motile population was accounted for in the CPC calculation (Fig. 6). It has been described for Escherichia coli that an $m s b B(l p x M)$ deletion results in outer membrane stress and $\sigma^{\mathrm{E}}$ release (Tam and Missiakas, 2005). In Salmonella, $\sigma^{\mathrm{E}}$ regulates 62 genes, governing roles in pathogenicity, oxidative stress resistance, and stationary-phase survival (Skovierova et al., 2006). It is therefore feasible to conceive that global regulatory changes are occurring upon restoring $m s b B$ in VNP20009 $c h e Y^{+}$, which could affect the motility of a population.

Our results revealed that complete restoration of VNP20009 chemotaxis cannot be achieved by replacing mutated genes of $c h e Y$ and $m s b B$ with their parental copies. However, significant increases in overall performance can be obtained. Although the lipid A modification by MsbB is essential for attenuation of VNP20009, it is not ideal for the downstream application of exploring the role of bacterial chemotaxis in tumor colonization. Research questions on utilizing bacterial chemotaxis for improved tumor targeting might be better suited in strains that have been attenuated by other means, such as auxotrophy or modifications of LPS with a less detrimental effect as described by Frahm et al. (Frahm et al., 2015). However, gene targets for auxotrophy should be chosen carefully. An $S$. Typhimurium aroA deletion strain, which is auxotroph for aromatic amino acids, was reported to exhibit altered flagellar phase variation (Felgner et al., 2016). It has already been suspected that VNP20009 is over-attenuated due to its lipid A modification, which could prevent a maintained, high cytokine TNF- $\alpha$ release or induction of an inflammatory response. Depletion of TNF- $\alpha$ has been shown to retard tumor blood influx by the vasculature and delay bacterial tumor colonization (Leschner et al., 2009). Together, these results might explain the Phase 1 Clinical Trail outcome using VNP20009 (Leschner et al., 2009), where significantly elevated TNF- $\alpha$ concentrations were detected in peripheral blood, however high enough levels were not maintained over the course of the study (Toso et al., 2002).

We discovered VNP20009 $\mathrm{cheY}^{+}$to have reduced chemotaxis compared to the parental strain. The reduction is at least in part due to the disruption of $m s b B$, the gene providing VNP20009 with an attenuated state. Prior to the present study, MsbB function has not been associated with chemotactic performance. Our objective was to maximize chemotaxis, in hopes of testing VNP20009 $\mathrm{cheY}^{+}$for future efficacy as a biotherapeutic. We achieved this goal without manipulating any characteristic features of VNP20009, in particular tumor targeting and its safety profile. Future studies utilizing VNP20009 $\mathrm{cheY}^{+}$will bear in mind potentially unknown phenotypes associated with not only the $m s b B$ encoded lipid A modification, but other genomic alterations present in the strain, which have given it the reputation as an anticancer therapeutic.

\section{Acknowledgments}

This study was supported by seed funds from the Fralin Life Science Institute at Virginia Tech to Birgit E. Scharf and National Science Foundation CAREER award (CBET-1454226) to Bahareh Behkam. We are grateful to Timofey Arapov for his assistance in fluorescent image acquisition and processing.

\section{Appendix A. Supplementary data}

Supplementary data associated with this article can be found, in the online version, at http://dx.doi.org/10.1016/j.jbiotec.2017.04.006.

\section{References}

al-Ramadi, B.K., Fernandez-Cabezudo, M.J., El-Hasasna, H., Al-Salam, S., Bashir, G., Chouaib, S., 2009. Potent anti-tumor activity of systemically-administered IL2expressing Salmonella correlates with decreased angiogenesis and enhanced tumor apoptosis. Clin. Immunol. 130, 89-97.

Attmannspacher, U., Scharf, B.E., Harshey, R.M., 2008. FliL is essential for swarming: motor rotation in absence of FliL fractures the flagellar rod in swarmer cells of Salmonella enterica. Mol. Microbiol. 68, 328-341.

Berger, E., Soldati, R., Huebener, N., Hohn, O., Stermann, A., Durmus, T., Lobitz, S. Zenclussen, A.C., Christiansen, H., Lode, H.N., Fest, S., 2013. Salmonella SL7207 application is the most effective DNA vaccine delivery method for successful tumor eradication in a murine model for neuroblastoma. Cancer Lett. 331, 167-173.

Bertani, G., 1951. Studies on lysogenesis. I. The mode of phage liberation by lysogenic Escherichia coli. J. Bacteriol. 62, 293-300.

Broadway, K.M., Modise, T., Jensen, R.V., Scharf, B.E., 2014. Complete genome sequence of Salmonella enterica serovar Typhimurium VNP20009, a strain engineered for tumor targeting. J. Biotechnol. 192 (Pt A), 177-178.

Broadway, K.M., Denson, E.A., Jensen, R.V., Scharf, B.E., 2015. Rescuing chemotaxis of the anticancer agent Salmonella enterica serovar Typhimurium VNP20009. J. Biotechnol. 211, 117-120. 
Chirullo, B., Ammendola, S., Leonardi, L., Falcini, R., Petrucci, P., Pistoia, C., Vendetti, S., Battistoni, A., Pasquali, P., 2015. Attenuated mutant strain of Salmonella Typhimurium lacking the ZnuABC transporter contrasts tumor growth promoting anti-cancer immune response. Oncotarget 6, 17648-17660.

Chorobik, P., Czaplicki, D., Ossysek, K., Bereta, J., 2013. Salmonella and cancer: from pathogens to therapeutics. Acta Biochim. Pol. 60, 285-297.

Clairmont, C., Lee, K.C., Pike, J., Ittensohn, M., Low, K.B., Pawelek, J., Bermudes, D., Brecher, S.M., Margitich, D., Turnier, J., Li, Z., Luo, X., King, I., Zheng, L.M., 2000 Biodistribution and genetic stability of the novel antitumor agent VNP20009, a genetically modified strain of Salmonella typhimurium. J. Infect. Dis. 181, 1996-2002.

Crull, K., Bumann, D., Weiss, S., 2011a. Influence of infection route and virulence factors on colonization of solid tumors by Salmonella enterica serovar Typhimurium. FEMS Immunol. Med. Microbiol. 62, 75-83.

Crull, K., Rohde, M., Westphal, K., Loessner, H., Wolf, K., Felipe-Lopez, A., Hensel, M., Weiss, S., 2011b. Biofilm formation by Salmonella enterica serovar Typhimurium colonizing solid tumours. Cellular Microbiol.V 13 1223-1233.

Datsenko, K.A., Wanner, B.L., 2000. One-step inactivation of chromosomal genes in Escherichia coli K-12 using PCR products. Proc. Natl. Acad. Sci. U.S.A 97, 6640-6645.

Eisenstark, A., Kazmierczak, R.A., Dino, A., Khreis, R., Newman, D., Schatten, H., 2007. Development of Salmonella strains as cancer therapy agents and testing in tumor cell lines. Methods in Mol. Biol.V 394 323-354.

Felgner, S., Frahm, M., Kocijanci, D., Rohde, M., Eckweiler, D., Bielecka, A., Bueno, E., Cava, F., Abraham, W., Curtiss, R.r., Häussler, S., Erhardt, M., Weiss, S., 2016. aroAdeficient Salmonella enterica serovar Typhimurium is more than a metabolically attenuated mutant. MBio 7 e01220-01216.

Forbes, N.S., 2010. Engineering the perfect (bacterial) cancer therapy. Nat. Rev. Cancer $10,785-794$.

Frahm, M., Felgner, S., Kocijancic, D., Rohde, M., Hensel, M., Curtiss III, R., Erhardt, M., Weiss, S., 2015. Efficiency of conditionally attenuated Salmonella enterica serovar Typhimurium in bacterium-mediated tumor therapy. MBio 6 e00254-00215.

Grille, S., Moreno, M., Bascuas, T., Marques, J.M., Munoz, N., Lens, D., Chabalgoity, J.A., 2014. Salmonella enterica serovar Typhimurium immunotherapy for B-cell lymphoma induces broad anti-tumour immunity with therapeutic effect. Immunology 143, $428-437$.

Hiroshima, Y., Zhao, M., Zhang, Y., Maawy, A., Hassanein, M.K., Uehara, F., Miwa, S., Yano, S., Momiyama, M., Suetsugu, A., Chishima, T., Tanaka, K., Bouvet, M., Endo, I., Hoffman, R.M., 2013. Comparison of efficacy of Salmonella typhimurium A1-R and chemotherapy on stem-like and non-stem human pancreatic cancer cells. Cell Cycle $12,2774-2780$.

Hiroshima, Y., Zhang, Y., Murakami, T., Maawy, A., Miwa, S., Yamamoto, M., Yano, S., Sato, S., Momiyama, M., Mori, R., Matsuyama, R., Chishima, T., Tanaka, K., Ichikawa, Y., Bouvet, M., Endo, I., Zhao, M., Hoffman, R.M., 2014. Efficacy of tumortargeting Salmonella typhimurium A1-R in combination with anti-angiogenesis therapy on a pancreatic cancer patient-derived orthotopic xenograft (PDOX) and cell line mouse models. Oncotarget 5, 12346-12357.

Hoffman, R.M., 2016. Tumor-targeting Salmonella typhimurium A1-R: An overview. Methods Mol. Biol. 1409, 1-8.

https://www.cancer.gov/publications/dictionaries/cancer-drug?cdrid $=37800$.

Jarosz, M., Jazowiecka-Rakus, J., Cichon, T., Glowala-Kosinska, M., Smolarczyk, R., Smagur, A., Malina, S., Sochanik, A., Szala, S., 2013. Therapeutic antitumor potential of endoglin-based DNA vaccine combined with immunomodulatory agents. Gene Ther. 20, 262-273.

Jia, H., Li, Y., Zhao, T., Li, X., Hu, J., Yin, D., Guo, B., Kopecko, D.J., Zhao, X., Zhang, L., Xu, D.Q., 2012. Antitumor effects of Stat3-siRNA and endostatin combined therapies, delivered by attenuated Salmonella, on orthotopically implanted hepatocarcinoma. Cancer Immunol. Immunother. 61, 1977-1987.

Jiang, S.N., Park, S.H., Lee, H.J., Zheng, J.H., Kim, H.S., Bom, H.S., Hong, Y., Szardenings, M., Shin, M.G., Kim, S.C., Ntziachristos, V., Choy, H.E., Min, J.J., 2013. Engineering of bacteria for the visualization of targeted delivery of a cytolytic anticancer agent. Mol. Ther. 21, 1985-1995.

Karlinsey, J.E., 2007. lambda-Red genetic engineering in Salmonella enterica serovar Typhimurium. Methods Enzymol. 421, 199-209.

Kasinskas, R.W., Forbes, N.S., 2006. Salmonella typhimurium specifically chemotax and proliferate in heterogeneous tumor tissue in vitro. Biotechnol. Bioeng. 94, 710-721.

Kasinskas, R.W., Forbes, N.S., 2007. Salmonella typhimurium lacking ribose chemoreceptors localize in tumor quiescence and induce apoptosis. Cancer Res. 67, 3201-3209.

Kawaguchi, K., Igarashi, K., Murakami, T., Chmielowski, B., Kiyuna, T., Zhao, M., Zhang, Y., Singh, A., Unno, M., Nelson, S.D., Russell, T.A., Dry, S.M., Li, Y., Eilber, F.C., Hoffman, R.M., 2016. Tumor-targeting Salmonella typhimurium A1-R combined with temozolomide regresses malignant melanoma with a BRAF-V600E mutation in a patient-derived orthotopic xenograft (PDOX) model. Oncotarget 7, 85929-85936.

Kim, J.E., Phan, T.X., Nguyen, V.H., Dinh-Vu, H.V., Zheng, J.H., Yun, M., Park, S.G., Hong, Y., Choy, H.E., Szardenings, M., Hwang, W., Park, J.A., Park, S., Im, S.H., Min, J.J., 2015. Salmonella typhimurium suppresses tumor growth via the pro-inflammatory cytokine interleukin-1beta. Theranostics 5, 1328-1342.

Lee, K.C., Zheng, L.-M., Luo, X., Clairmont, C., Fischer, J., Margitich, D., Turnier, J., Almassian, B., Bermudes, D., King, I., 2000. Comparative evaluation of the acute toxic effects in monkeys, pigs and mice of a genetically engineered Salmonella strain (VNP20009) being developed as an antitumor agent. Int. J. Toxicol. 19, 19-25.

Lee, S.H., Mizutani, N., Mizutani, M., Luo, Y., Zhou, H., Kaplan, C., Kim, S.W., Xiang, R., Reisfeld, R.A., 2006. Endoglin (CD105) is a target for an oral DNA vaccine against breast cancer. Cancer Immunol. Immunother. 55, 1565-1574.

Leschner, S., Weiss, S., 2010a. Salmonella-allies in the fight against cancer. Journal of Molecular Medicine 88, 763-773.
Leschner, S., Weiss, S., 2010b. Salmonella-allies in the fight against cancer. J. Mol. Med. (Berlin, Germany) 88, 763-773.

Leschner, S., Westphal, K., Dietrich, N., Viegas, N., Jablonska, J., Lyszkiewicz, M., Lienenklaus, S., Falk, W., Gekara, N., Loessner, H., Weiss, S., 2009. Tumor invasion of Salmonella enterica serovar Typhimurium is accompanied by strong hemorrhage promoted by TNF-alpha. PLoS ONE 4, e6692.

Li, Z., Yin, P.H., Yang, S.S., Li, Q.Y., Chang, T., Fang, L., Shi, L.X., Fang, G.E., 2013. Recombinant attenuated Salmonella typhimurium carrying a plasmid co-expressing ENDO-VEGI151 and survivin siRNA inhibits the growth of breast cancer in vivo. Mol. Med. Rep. 7, 1215-1222.

Li, C.X., Yu, B., Shi, L., Geng, W., Lin, Q.B., Ling, C.C., Yang, M., Ng, K.T., Huang, J.D., Man, K., 2017. 'Obligate' anaerobic Salmonella strain YB1 suppresses liver tumor growth and metastasis in nude mice. Oncol. Lett. 13, 177-183.

Lin, C.S., Kao, S.H., Chen, Y.C., Li, C.H., Hsieh, Y.T., Yang, S.C., Wu, C.J., Lee, R.P., Liao, K.W., 2012. Enhancement of anti-murine colon cancer immunity by fusion of a SARS fragment to a low-immunogenic carcinoembryonic antigen. Biol. Proced. Online $14,2$.

Low, K.B., Ittensohn, M., Luo, X., Zheng, L.M., King, I., Pawelek, J.M., Bermudes, D., 2004. Construction of VNP20009: a novel, genetically stable antibiotic-sensitive strain of tumor-targeting Salmonella for parenteral administration in humans. Methods Mol. Med. 90, 47-60.

Low, K.B., Murray, S.R., Pawelek, J., Bermudes, D., 2016. Isolation and analysis of suppressor mutations in tumor-targeted msbB Salmonella. Methods Mol. Biol. 1409, 95-123.

Manuel, E.R., Blache, C.A., Paquette, R., Kaltcheva, T.I., Ishizaki, H., Ellenhorn, J.D., Hensel, M., Metelitsa, L., Diamond, D.J., 2011. Enhancement of cancer vaccine therapy by systemic delivery of a tumor-targeting Salmonella-based STAT3 shRNA suppresses the growth of established melanoma tumors. Cancer Res. 71, 4183-4191.

Murakami, T., DeLong, J., Eilber, F.C., Zhao, M., Zhang, Y., Zhang, N., Singh, A., Russell, T., Deng, S., Reynoso, J., Quan, C., Hiroshima, Y., Matsuyama, R., Chishima, T., Tanaka, K., Bouvet, M., Chawla, S., Endo, I., Hoffman, R.M., 2016. Tumor-targeting Salmonella typhimurium A1-R in combination with doxorubicin eradicate soft tissue sarcoma in a patient-derived orthotopic xenograft (PDOX) model. Oncotarget 7, 12783-12790.

Murray, S.R., Bermudes, D., de Felipe, K.S., Low, K.B., 2001. Extragenic suppressors of growth defects in msbB Salmonella. J. Bacteriol. 183, 5554-5561.

Murray, S.R., de Felipe, K.S., Obuchowski, P.L., Pike, J., Bermudes, D., Low, K.B., 2004 Hot spot for a large deletion in the 18- to 19-centisome region confers a multiple phenotype in Salmonella enterica serovar Typhimurium strain ATCC 14028. J. Bacteriol. 186, 8516-8523.

Nallar, S.C., Xu, D.Q., Kalvakolanu, D.V., 2017. Bacteria and genetically modified bacteria as cancer therapeutics: Current advances and challenges. Cytokine 89, 160-172.

\section{NCT01099631, C. t. I.}

Nguyen, V.H., Kim, H.S., Ha, J.M., Hong, Y., Choy, H.E., Min, J.J., 2010. Genetically engineered Salmonella typhimurium as an imageable therapeutic probe for cancer. Cancer Res. 70, 18-23.

Pawelek, J.M., Low, K.B., Bermudes, D., 1997. Tumor-targeted Salmonella as a novel anticancer vector. Cancer Res. 57, 4537-4544.

Pawelek, J.M., Low, K.B., Bermudes, D., 2003. Bacteria as tumour-targeting vectors. The Lancet Oncology 4, 548-556.

Qian, B.J., Yan, F., Li, N., Liu, Q.L., Lin, Y.H., Liu, C.M., Luo, Y.P., Guo, F., Li, H.Z., 2011 MTDH/AEG-1-based DNA vaccine suppresses lung metastasis and enhances chemosensitivity to doxorubicin in breast cancer. Cancer Immunol. Immunother. 60, 883-893.

Roider, E., Jellbauer, S., Kohn, B., Berchtold, C., Partilla, M., Busch, D.H., Russmann, H., Panthel, K., 2011. Invasion and destruction of a murine fibrosarcoma by Salmonellainduced effector CD8 T cells as a therapeutic intervention against cancer. Cancer Immunol. Immunother. 60, 371-380.

Saltzman, D.A., Heise, C.P., Hasz, D.E., Zebede, M., Kelly, S.M., Curtiss III, R., Leonard, A.S., Anderson, P.M., 1996. Attenuated Salmonella typhimurium containing interleukin-2 decreases MC-38 hepatic metastases: a novel anti-tumor agent. Cancer Biother. Radiopharm. 11, 145-153.

Shi, L., Yu, B., Cai, C.H., Huang, W., Zheng, B.J., Smith, D.K., Huang, J.D., 2016. Combined prokaryotic-eukaryotic delivery and expression of therapeutic factors through a primed autocatalytic positive-feedback loop. J. Control Release 222, 130-140.

Silva-Valenzuela, C.A., Desai, P.T., Molina-Quiroz, R.C., Pezoa, D., Zhang, Y., Porwollik, S., Zhao, M., Hoffman, R.M., Contreras, I., Santiviago, C.A., McClelland, M., 2016. Solid tumors provide niche-specific conditions that lead to preferential growth of Salmonella. Oncotarget 7, 35169-35180.

Skovierova, H., Rowley, G., Rezuchova, B., Homerova, D., Lewis, C., Roberts, M., Kormanec, J., 2006. Identification of the sigmaE regulon of Salmonella enterica serovar Typhimurium. Microbiology 152, 1347-1359.

Somerville Jr., J.E., Cassiano, L., Bainbridge, B., Cunningham, M.D., Darveau, R.P., 1996. A novel Escherichia coli lipid A mutant that produces an antiinflammatory lipopolysaccharide. J. Clin. Invest. 97, 359-365.

Stritzker, J., Weibel, S., Seubert, C., Gotz, A., Tresch, A., van Rooijen, N., Oelschlaeger, T.A., Hill, P.J., Gentschev, I., Szalay, A.A., 2010. Enterobacterial tumor colonization in mice depends on bacterial metabolism and macrophages but is independent of chemotaxis and motility. Int. J. Med. Microbiol.: IJMM 300, 449-456.

Tam, C., Missiakas, D., 2005. Changes in lipopolysaccharide structure induce the sigma (E)-dependent response of Escherichia coli. Mol. Microbiol. 55, 1403-1412.

Thamm, D.H., Kurzman, I.D., King, I., Li, Z., Sznol, M., Dubielzig, R.R., Vail, D.M., MacEwen, E.G., 2005. Systemic administration of an attenuated, tumor-targeting Salmonella typhimurium to dogs with spontaneous neoplasia: phase I evaluation. Clin. 
Cancer Res: An Official Journal of the American Association for Cancer Research 11, 4827-4834.

Toso, J.F., Gill, V.J., Hwu, P., Marincola, F.M., Restifo, N.P., Schwartzentruber, D.J., Sherry, R.M., Topalian, S.L., Yang, J.C., Stock, F., Freezer, L.J., Morton, K.E., Seipp, C., Haworth, L., Mavroukakis, S., White, D., MacDonald, S., Mao, J., Sznol, M., Rosenberg, S.A., 2002. Phase I study of the intravenous administration of attenuated Salmonella typhimurium to patients with metastatic melanoma. J. Clin. Oncol. 20, 142-152.

Traore, M.A., Behkam, B., 2013. A PEG-DA microfluidic device for chemotaxis studies. J. Micromech. Microeng. 23, 85014.

Turner, L., Ryu, W.S., Berg, H.C., 2000. Real-time imaging of fluorescent flagellar filaments. J. Bacteriol. 182, 2793-2801.

VanCott, J.L., Chatfield, S.N., Roberts, M., Hone, D.M., Hohmann, E.L., Pascual, D.W., Yamamoto, M., Kiyono, H., McGhee, J.R., 1998. Regulation of host immune responses by modification of Salmonella virulence genes. Nat. Med. 4, 1247-1252.

Xiang, R., Mizutani, N., Luo, Y., Chiodoni, C., Zhou, H., Mizutani, M., Ba, Y., Becker, J.C., Reisfeld, R.A., 2005. A DNA vaccine targeting survivin combines apoptosis with suppression of angiogenesis in lung tumor eradication. Cancer Res. 65, 553-561.

Xiong, G., Husseiny, M.I., Song, L., Erdreich-Epstein, A., Shackleford, G.M., Seeger, R.C., Jackel, D., Hensel, M., Metelitsa, L.S., 2010. Novel cancer vaccine based on genes of Salmonella pathogenicity island 2. Int. J. Cancer 126, 2622-2634.

Xu, X., Hegazy, W.A., Guo, L., Gao, X., Courtney, A.N., Kurbanov, S., Liu, D., Tian, G., Manuel, E.R., Diamond, D.J., Hensel, M., Metelitsa, L.S., 2014. Effective cancer vaccine platform based on attenuated Salmonella and a type III secretion system. Cancer Res. 74, 6260-6270.
Yano, S., Zhang, Y., Zhao, M., Hiroshima, Y., Miwa, S., Uehara, F., Kishimoto, H., Tazawa, H., Bouvet, M., Fujiwara, T., Hoffman, R.M., 2014. Tumor-targeting Salmonella typhimurium A1-R decoys quiescent cancer cells to cycle as visualized by FUCCI imaging and become sensitive to chemotherapy. Cell Cycle 13, 3958-3963.

Ye, J., Li, L., Zhang, Y., Zhang, X., Ren, D., Chen, W., 2013. Recombinant Salmonellabased 4-1BBL vaccine enhances T cell immunity and inhibits the development of colorectal cancer in rats: in vivo effects of vaccine containing 4-1BBL. J. Biomed. Sci. $20,8$.

Yoon, W., Choi, J.H., Kim, S., Park, Y.K., 2014. Engineered Salmonella typhimurium expressing E7 fusion protein, derived from human papillomavirus, inhibits tumor growth in cervical tumor-bearing mice. Biotechnol. Lett. 36, 349-356.

Yu, B., Shi, L., Zhang, B.Z., Zhang, K.E., Peng, X., Niu, H.B., Qu, J.L., 2015. Obligate anaerobic Salmonella typhimurium strain YB1 treatment on xenograft tumor in immunocompetent mouse model. Oncol. Lett. 10, 1069-1074.

Zhang, L., Gao, L., Zhao, L., Guo, B., Ji, K., Tian, Y., Wang, J., Yu, H., Hu, J., Kalvakolanu, D.V., Kopecko, D.J., Zhao, X., Xu, D.Q., 2007. Intratumoral delivery and suppression of prostate tumor growth by attenuated Salmonella enterica serovar typhimurium carrying plasmid-based small interfering RNAs. Cancer Res. 67, 5859-5864.

Zhao, M., Yang, M., Li, X.M., Jiang, P., Baranov, E., Li, S., Xu, M., Penman, S., Hoffman, R.M., 2005. Tumor-targeting bacterial therapy with amino acid auxotrophs of GFPexpressing Salmonella typhimurium. Proc. Natl. Acad. Sci. U.S.A. 102, 755-760.

Zhao, M., Geller, J., Ma, H., Yang, M., Penman, S., Hoffman, R.M., 2007. Monotherapy with a tumor-targeting mutant of Salmonella typhimurium cures orthotopic metastatic mouse models of human prostate cancer. Proc. Natl. Acad. Sci. U.S.A. 104, 10170-10174. 